\title{
Osteopathic manipulation for pubic symphysis dysfunction during spontaneous labor: a case study
}

\begin{abstract}
Pubic symphysis dysfunction is a common condition in pregnancy. Most pubic symphysis dysfunction involves a vertical shear, is minor, and is easily treated with simple osteopathic manipulation techniques. The obstetrician (OB) hospitalist was an osteopathic physician, and requested permission to treat the patient from the attending physician with osteopathic manipulation which was approved. Direct myofasical release was used to restore equal range of motion of the sacroiliac joints, then was used to correct restrictions in the suprapubic fascia. Symmetry of the pelvic structures was restored, the patient reported a significant decrease in pain, and was able to lift her feet when ambulating.
\end{abstract}

Volume 5 Issue 2 - 2017

\author{
Showalter A, McCarroll ML \\ Department of Clinical Medicine, Pacific Northwest University, \\ USA \\ Correspondence: Michele L McCarroll, Ph.D, CCRP,ACSM- \\ CCEP, FAACVPR, Chief Research Officer \\ Professor of Clinical Medicine, College of Osteopathic Medicine, \\ Pacific Northwest University of Health Sciences, Office of \\ Scholarly Activity, Iron Horse Lodge 2nd Floor, I I I University \\ Parkway, Suite 202, Yakima,WA 9890I, USA, Tel 509249 7730, \\ Email mmccarroll@pnwu.edu
}

Received: June 07, 2017 | Published: June 16, 2017

\section{Introduction}

Pubic symphysis dysfunction is a common condition in pregnancy. ${ }^{1,2}$ The hormone relaxin has a primary softening effect on the symphyses. ${ }^{3,4}$ This, in combination with the center of gravity changes of the enlarging uterus, causes strain patterns in the ligamentous tissues that the patient may not be able to overcome spontaneously. ${ }^{5}$ Most pubic symphysis dysfunction involves a vertical shear, is minor, ${ }^{6}$ and is easily treated with simple osteopathic manipulation techniques. ${ }^{7-9}$ Anterior/posterior shears are more rare, but cause much more pain and disability. ${ }^{10}$ Additionally, significant trauma due to the shears is often in the patient history.

\section{Case report}

The patient, at term, was noted to be 4 centimeters (CM) dilated, $90 \%$ effaced and 0 station with regular painful contractions. In addition, she had been having pubic symphysis pain for several months that had not been treated. In addition to pelvic pain, she had difficulty with normal activities such as walking and lifting her feet. Her physician was notified of admission in spontaneous labor. The obstetrician (OB) hospitalist was an osteopathic physician, and requested permission to treat the patient from the attending physician with osteopathic manipulation which was approved. The patient was verbally consented for the procedure. Structural examination revealed an exquisitely tender pubic symphysis with both vertical and anterior posterior shears noted. There were restrictions in range of motion of the sacroiliac joints bilaterally, worse on the patient's right. Asymmetry of the anterior superior iliac spines was also noted. Muscle energy technique was used to correct the anterior/posterior pubic shear, and a leg tug was used to correct the vertical component Direct myofasical release was used to restore equal range of motion of the sacroiliac joints, then was used to correct restrictions in the suprapubic fascia. Symmetry of the pelvic structures was restored, the patient reported a significant decrease in pain, and was able to lift her feet when ambulating. She was advised to seek osteopathic treatment after the delivery, as there is often an exacerbation of symptoms because of the stress on the symphysis with vaginal delivery. Her physician was advised to order a trochanter belt for the patient after delivery for pelvic stabilization.

The patient received epidural anesthesia and successfully completed a vaginal delivery. After delivery, the patient was being assisted to move in bed before full sensation had returned to her legs, and one leg inadvertently dropped off the bed. The patient immediately felt her symptoms return. There was no one available at the time to give treatment. An abdominal binder rather than a trochanter belt was ordered which did not provide relief to the patient. After discharge, she used a walker belonging to an elderly relative for ambulation. Five days after the delivery, still in significant pain and unable to ambulated independently, she contacted the hospitalist who had treated her for assistance in finding follow up.

\section{Discussion}

Osteopathic medical students are well versed in vertical pubic shear, although anterior/posterior shears, being much less common, may go unrecognized. Allopathic physicians may not be familiar with pubic shear, so symptoms may be regarded as part of usual discomforts of pregnancy. If recognized, the physician may not be aware of treatments available or may be reluctant to refer for osteopathic care.

\section{Signs and symptoms}

Patients with mild pubic shear may report a pinchy pain in the anterior mid pelvis that is exacerbated by ambulation. ${ }^{11}$ The pain is constant, and when presenting to triage for evaluation, the constant nature of the pain distinguishes pubic shear from signs and symptoms of labor. History may include pain beginning when arising from a seated position or getting out of bed. It may occur with bending and twisting, getting into a car one leg first or arising from a seated position with one leg in front of the other. Anterior/posterior shear causes deep severe pelvic pain, as the pelvic diaphragm has unequal tensions from side to side. The patient may have history of trauma such a motor vehicle accident, a fall or obstetric injury. A shear may be induced at the time of delivery if the patient has regional anesthesia 
and during second stage unequal pressures are placed on the patient's legs while assisting her. The symphysis will be exquisitely tender on the side of dysfunction.

\section{Structural exam}

Osteopathic physicians diagnosis somatic dysfunction with tenderness, asymmetry, restrictionof motion, and tissue texture changes (TART) findings. Any of the following findings in tissue is indicative of somatic dysfunction:
i. Tenderness $(\mathrm{T})$,
ii. Asymmetry (A),
iii. Restriction of motion $(\mathrm{R})$,
iv. Tissue texture changes (T).

The structural examination of the pelvis should include evaluation of the anterior superior iliac spines for symmetry. The pubic symphysis is then palpated for tenderness, and thumbs placed on the superior border of the pubic bones will show whether one side is more superior to the other, indicative of a vertical shear. The corresponding anterior superior iliac spine will also be superior in vertical pubic shear. To evaluate for anterior/posterior shear, thumbs are placed on the anterior surface of the pubic symphysis equidistance from the midline. Again, symmetry or asymmetry is determined. If an anterior/posterior shear is present, one side will be more posterior, and the side of dysfunction will be exquisitely tender. Testing sacroiliac dysfunction can be done by using compression of the iliac crest into the sacroiliac joint. Normally there is a subtle springing, or giving way with compression. If one side does not spring, that is the side of dysfunction. The sacrum may also be checked for sacral torsion or other dysfunction.

\section{Treatment}

Osteopathic techniques are applied to correct TART findings with the goal of restoring full range of motion, symmetry and relieving pain. The specific treatments applied to this case will be discussed. When a significant dysfunction such as anterior/posterior shear is encountered, it is often necessary to correct some of the surrounding dysfunction before the primary problem is addressed. In this case, the anterior superior iliac spine asymmetry was treated with facilitated positioning. The patient was placed with legs in a figure of four fashion, straight leg on the more inferior side. The operator places sufficient downward force on the bent knee so the patient must engage pelvic muscles to straighten her leg. This rotates the iliac crest of the straight leg posterior, also elevating the pubic symphysis and partially corrected the vertical shear. A gentle leg tug was then done on the superior side to complete treatment of the vertical shear.

Muscle energy techniques also utilize patient muscle engagement to create a change in the target tissue. With the patient in semiFowler's position, the knee of the posterior pubic symphysis was bent and internally rotated to come past midline until resistance was encountered. The patient was asked to gently push against the operator's resistance in external rotation. When the patient relaxes, the leg may be passively moved to a new position of ease. This is repeated several times, progressively increasing internal rotation of the extremity and altering muscle tension. The last time, the patient's leg is passively straightened while keeping it in internal rotation. The muscle engagement necessary for this activity also brings the pubic symphysis on that side more anterior. The symphysis is rechecked and the procedure repeated if necessary. The effect of an anterior/posterior shear, in addition to pelvic floor dysfunction, includes strain patterns in the suprapubic tissues. After treatment of the shear, myofasical release treatment of the suprapubic tissues first is very tender, but after a few minutes provides significant relief of discomfort. The patient's sacroiliac dysfunction was treated with a passive full range of motion of the joint. It is important to address all identified somatic dysfunction with anterior/posterior shear to provide tissue stability and prevent recurrence of the dysfunction. ${ }^{12}$ Long-standing dysfunction does tend to recur, but the more thorough the treatment, the longer it takes to recur. Furthermore, with each successful treatment, and with proper exercise of the patient, the positive treatment effect will be longer and longer until resolved. Treatment dosing intervals are aimed at treating again when symptoms begin to recur and before significant decompensation has occurred.

\section{Osteopathic postpartum management}

The astute osteopathic physician will be watchful for signs of somatic dysfunction immediately following delivery. ${ }^{13}$ While there is more potential trauma after vaginal delivery, relax in still is present after cesarean delivery, and the patient being moved while under anesthesia can induce somatic dysfunction that may not have been present before delivery. ${ }^{3,4}$ Symptoms after delivery indicative of pelvic somatic dysfunction include mid-pelvic anterior pain, difficulty weight bearing or moving in bed, and pain with ambulation. One of the most significant injuries is pubic symphysis diaphysis. In this case, some or all of the ligamentous fibers of the symphysis are disrupted. It can be diagnosed by x-ray evidence of abnormal widening of the symphysis. The patient may not be able to ambulate, therefore at higher risk for deep vein thrombosis. Standard treatment is to give steroids or anti-inflammatories and a walker for ambulation. Without appropriate physical therapy and/or osteopathic treatment, disability and dysfunction may not resolve. After delivery, especially if anesthesia is used, care should be taken to move the extremities symmetrically. Osteopathic physicians often will take the legs and do a range of motion in internal rotation to "set" the pelvis after delivery. If the patient has any signs or symptoms of dysfunction, gentle treatment should be initiated daily while an inpatient. ${ }^{14}$ A trochanter belt may be placed after symmetry is resorted to provide additional support while relaxin begins to subside. The trochanter belt is placed over the buttocks, trochanter and pubic symphysis to give needed support. Sacroiliac belts are more common, but do not apply pressure correctly for this purpose. Abdominal binders are too stretchy to work, even if placed over the appropriate areas. Patients have much greater ease of ambulation with the trochanter belt. If a trochanter belt is not available a homemade belt of cotton denim or duck can be made by cutting a two inch strip of fabric and tying it over the same points. Follow up should be two to three times weekly during the first postpartum week depending on the severity of injury, and continued at least through the six week postpartum period while relax in is dissipating. ${ }^{8} 12$ Treatment intervals are extended as the patient improves. Treating early at appropriate intervals reduces that chance the patient will have long-term pain and disability.

\section{Patient education}

Patients should be given instructions on how to do daily activities without further injury or strain on the symphysis. Teach the patient to get out of bed by rolling on the side with knees bent, dropping the legs off the bed while pushing the trunk of the body upright with the hands. This provides a method of getting out of bed without twisting 
the pelvis. When rising from a seated position, the feet should be together, not one in front of the other. When sitting in a car, do it "princess style". With feet on the ground, facing away from the car, sit down on the seat. Legs are then rotated into the car together. When picking up objects off the ground, use a squat rather than bend and twist. These simple instructions will help prevent re-injury and aid the healing process.

\section{Appropriate treatment and referral}

As in all things pertaining to health, prevention is the best cure. ${ }^{15}$ Osteopathic treatment during prenatal care has been shown in retrospective case control and randomized prospective studies to decrease disability in pregnancy. ${ }^{16-19}$ While it is common to have pelvic and back pain in pregnancy, it is not normal. When patients present with symptoms, screening for somatic dysfunction should be done and treatment or referral offered. The Accreditation Council for Graduate Medical Education (ACGME) single accreditation system will provide new opportunities for allopathic physicians to become familiar with osteopathic diagnoses and learn osteopathic techniques if they so desire. These joint training opportunities will benefit patients when trainees become familiar with treatment options available. Prompt recognition and treatment of pelvic somatic dysfunction will reduce suffering on the part of our patients. ${ }^{12}$

\section{Acknowledgements}

None.

\section{Conflict of interest}

The author declares no conflict of interest

\section{References}

1. Albert H, Godskesen M, Westergaard J. Prognosis in four syndromes of pregnancy-related pelvic pain. Acta Obstet Gynecol Scand. 2001;80(6):505-510.

2. Hacker, Nevill F. Essentials of Obstetrics and Gynecology. 6th ed. USA: Elsevier; 2016. p. 62-63.

3. Goldsmith LT, Weis W. Relaxin in human pregnancy. Ann N Y Acad Sci. 2009;1160:130-135.

4. Aldabe D, Ribeiro DC, Milosavljevic S, et al. Pregnancy-related pelvic girdle pain and its relationship with relaxin levels during pregnancy: a systematic review. Eur Spine J. 2012;21(9):1769-1776.

5. Mens JM, Pool-Goudzwaard A, Stam HJ. Mobility of the pelvic joints in pregnancy-related lumbopelvic pain: a systematic review. Obstet Gynecol Surv. 2009;64(3):200-208.
6. Pires R, Labronici PJ, Giordano V, et al. Intrapartum pubic symphysis disruption. Ann Med Health Sci Res. 2015;5(6):476-479.

7. Still AT. The Philosophy and Mechanical Principles of Osteopathy. USA: Hudson-Kimberly Publishing Co; 1902. p. 1-324.

8. King HH. Osteopathic manipulative treatment in prenatal care: evidence supporting improved outcomes and health policy implications. AAOJ. 2000;10:25-33.

9. Hitchcock ME (1976) Osteopathic care in pregnancy. Osteopath Ann 4: 405-411.

10. Björklund K, Bergström S, Nordström ML, et al. Symphyseal distention in relation to serum relax in levels and pelvic pain in pregnancy. Acta Obstet Gynecol Scand. 2000;79(4):269-275.

11. Sipko T, Grygier D, Barczyk K, et al. The occurrence of strain symptoms in the lumbosacral region and pelvis during pregnancy and after childbirth. J Manipulative Physiol Ther. 2010;33(5):370-377.

12. Kenneth EN, Glonek T. Somatic dysfunction in osteopathic family medicine. american college of osteopathic family physicians. USA: Lippincott Williams and Wilkins; 2007. p. 1-532.

13. Russell R, Groves P, Taub N. Assessing long term backache after childbirth BMJ. 1993;306(6888):1299-1303.

14. Whiting L. Osteopathic prevention of certain complications of labor and the puerperium. JAOA. 1945;44:495-498.

15. Liddle SD, Pennick V. Interventions for preventing and treating lowback and pelvic pain during pregnancy. Cochrane Database Syst Rev. 2015;30(9):CD001139.

16. Hensel KL, Buchanan S, Brown SK, et al. Pregnancy research on osteopathic manipulation optimizing treatment effects: The PROMOTE study protocol. Am J Obstet Gynecol. 2016;116(11):716-724.

17. Schwerla F, Rother K, Rother D, et al. Osteopathic Manipulative Therapy in Women With Postpartum Low Back Pain and Disability: A Pragmatic Randomized Controlled Trial. J Am Osteopath Assoc. 2015;115(7):416425.

18. Licciardone JC, Buchanan S, Hensel KL, et al. Osteopathic manipulative treatment of back pain and related symptoms during pregnancy: a randomized controlled trial. Am J Obstet Gynecol. 2010;202(1):43.e1-43. e8.

19. King HH, Tettambel MA, Lockwood MD, et al. Osteopathic manipulative treatment in prenatal care: a retrospective case control design study. $J \mathrm{Am}$ Osteopath Assoc. 2003;103(12):577-582. 